\title{
Is Rome III Criteria for Irritable Bowel Syndrome More Useful Than Rome II Criteria in Korea?
}

TO THE EDITOR: Irritable bowel syndrome (IBS) is one of the most common disorders in primary care and gastroenterology practices. ${ }^{1}$ The prevalence of IBS varies widely from $3.3 \%$ to $12.1 \%$ with diagnostic criteria even in the same population. ${ }^{2} \mathrm{We}$ still do not know the true prevalence of IBS. Accurately determining the epidemiology of IBS is important to provide basis for assessing the burden of disease in socio-cultural, healthcare and economic terms. With great interest, I read the report by Park et $\mathrm{al}^{3}$ regarding the differences in prevalence and sociodemographic characteristics of irritable bowel syndrome according to Rome II and Rome III. In table 3, the authors presented a good agreement in the prevalence of IBS based on the diagnostic criteria. However a good agreement between Rome II and Rome III criteria in diagnosing IBS is inconsistent with the results of study by Sperber et al. ${ }^{4}$ A 3 to 4-fold of increase was observed in IBS prevalence with Rome III criteria (11.4\% vs $2.9 \%)$ in Sperber's study. Another interesting finding of this study was a higher prevalence of IBS by Rome II criteria compared with that of Sperber's study ( $8.0 \%$ vs $2.9 \%$ ). A possible explanation for the difference in the prevalence of IBS between 2 studies may be the low response rate in Park's study compared with that of Sperber's study $(26.2 \%$ vs $54.0 \%)$.

In table 5 , the authors showed the sociodemographic characteristics in group A, B and C. Group A was comprised of 16 subjects who met Rome II criteria but not Rome III and Group C was comprised of 26 subjects who met Rome III criteria but not Rome II. Sixty-five subjects in Group B met both Rome II and III criteria. Although the socioeconomic characteristics of the 3 groups were provided, there was no information regarding the psychological aspects. According to Sperber's study, individuals with Rome II criteria had more doctor visits, perception of stress and a negative global feeling. These findings suggest that Rome III criteria may better reflect the average IBS patient we see in our clinics. Clinical usefulness of Rome III criteria in IBS is still needed to be investigated.

Despite several limitations of the present study, it provides us with the important clues in understanding the epidemiological characteristics of Korean IBS patients based on Rome II and III criteria. Further large studies are needed to apply Rome III criteria widely to clinical practices and researches in Korea.

Moo In Park, MD

Department of Internal Medicine Kosin University College of Medicine Busan, Korea

1. Drossman DA, Camilleri M, Mayer EA, Whitehead WE. AGA technical review on irritable bowel syndrome. Gastroenterology 2002; 123:2108-2131.

2. Mearin F, Badía X, Balboa A, et al. Irritable bowel syndrome prevalence varies enormously depending on the employed diagnostic criteria: comparison of Rome II versus previous criteria in a general population. Scand J Gastroenterol 2001;36:1155-1161.

3. Park DW, Lee OY, Shim SG, et al. The differences in prevalence and sociodemographic characteristics of irritable bowel Syndrome according to Rome II and Rome III. J Neurogastroenterol Motil 2010;16:186-193.

4. Sperber AD, Shvartzman P, Friger M, Fich A. A comparative reappraisal of the Rome II and Rome III diagnostic criteria: are we getting closer to the 'true prevalence of irritable bowel syndrome? Eur J Gastroenterol Hepatol 2007;19:441-447.

\section{Conflicts of interest: None.}

